\title{
FADE DEPTH PREDICTION ON WIRELESS MICROWAVE LINKS USING TWO-RAY MULTIPATH MODEL ${ }^{1}$
}

\author{
S. Loyka, A. Kouki, F. Gagnon \\ Department of Electrical Engineering \\ Ecole de Technologie Superieure \\ 1100, Notre-Dame St. West \\ Montreal (Quebec), H3C 1K3, Canada \\ Email: sergey.loyka@ieee.org
}

\begin{abstract}
Fade depth prediction for airborne communication links is considered in this paper. There is no fading model for this specific scenario at the moment. The two closest models are the Olsen-Segal model for terrestrial links and the ITU-R model for satellite links. However, they cannot be directly applied to the airborne scenario. We propose a two-ray multipath fading model adapted to a realistic scenario of hilly or mountainous terrain, which applies to elevations angles higher than $2^{0}$ and frequencies lower than $10 \mathrm{GHz}$, when the contribution of ground multipath component is dominant. It is interesting to note that the two-ray model predicts roughly the same fade depth dependence on the path clearance angle as the OlsenSegal model, which may be considered as a theoretical justification, as to the best of our knowledge- for the first time, of the path elevation angle factor in that model. We further propose a hybrid approach to account for the atmospheric contribution to the total fade depth.
\end{abstract}

\section{INTRODUCTION}

Signal fading is a severe obstacle to reliable microwave communications. It affects the system performance in several ways. In this paper, we consider the impact of fading on signal-to-noise ratio (SNR) at the receiver of an ground-to-air (airborne) communication link. This effect is accounted for in the link power budget analysis using the fade margin, which quantifies the loss in SNR under fading conditions, usually - for a given fade outage probability (i.e., the probability that the given fade depth will be exceeded). The received signal power can be presented as $[1,2]$ :

\footnotetext{
${ }^{1}$ This work was supported in part by NSERC and BAE Systems Canada
}

$$
P_{R}=\frac{P_{T} G_{T} G_{R}}{L_{T} L_{R} L_{P}}
$$

where $\mathrm{P}_{R}$ and $\mathrm{P}_{T}$ are received and transmitted power correspondingly, $G_{R}$ and $G_{T}$ are receive and transmit antenna gains correspondingly, $\mathrm{L}_{\mathrm{R}}$ and $\mathrm{L}_{\mathrm{T}}$ are receive and transmit path losses (i.e., cable loss, loss due to aging, antenna misalignment etc.), and $\mathrm{L}_{\mathrm{P}}$ is propagation channel $\operatorname{loss}\left(L_{R}, L_{T}, L_{p}>1\right)$. The propagation loss is usually factored out into three main components $[3,4]$ :

$$
L_{P}=L_{0} L_{S} L_{L}
$$

where $\mathrm{L}_{0}$ is the average path loss (for example, free space loss), $\mathrm{L}_{\mathrm{L}}$ is the long-term fading (for example, due to shadowing), and $\mathrm{L}_{\mathrm{S}}$ is the short-term fading due to multipath. Hence, the fade margin can be defined as:

$$
F=L_{S} L_{L}
$$

The transmitter power or the transmit or receive antenna gains must be increased by $F$ to sustain the reliable link operation as compared to the case of unfaded propagation channel. The main concern for line-of-sight (LOS) links operating at less than $10 \mathrm{GHz}$ is the sort-term fading, which may be produced by ground or atmospheric multipath (however, other atmospheric impairments may sometime contribute as well, especially at low clearance angle).

An accurate estimation of fade depth is of great importance for the design of highly reliable communication link. Most models for signal fading prediction that are used in microwave wireless link budged analysis are mainly empirical in nature and rely on extensive measured data. Consequently, these models include the aggregate effects of the different fading 
mechanisms and do not allow for any insight into the relative importance of these mechanisms. When theoretical models are proposed, they are often limited to particular fading scenarios and are useful when such scenarios represent the dominant mechanism of fading. When the microwave wireless link considered is covered by an existing empirical model, that model is used and the relative contribution of different fading mechanisms is not important. However, if the link considered is not covered by any existing empirical model, additional analysis of the relative contribution of different fading mechanisms is of large importance.

\section{AIRBORNE LINK SCENARIO}

In this paper, we consider the airborne link scenario shown on Fig. 1. In this scenario, the ground station 1 communicates with the ground station 2 through the airborne repeater node.

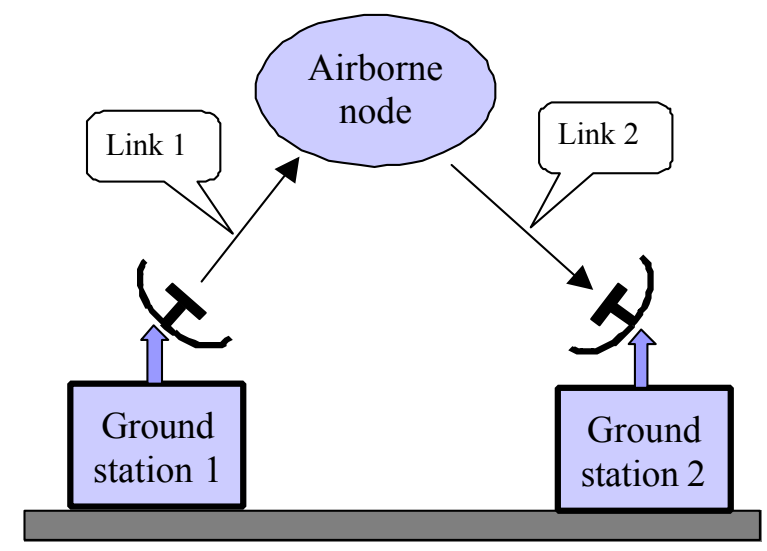

Figure 1. Airborne communication link scenario. Ground station 1 communicates with ground station 2 using the airborne repeater node.

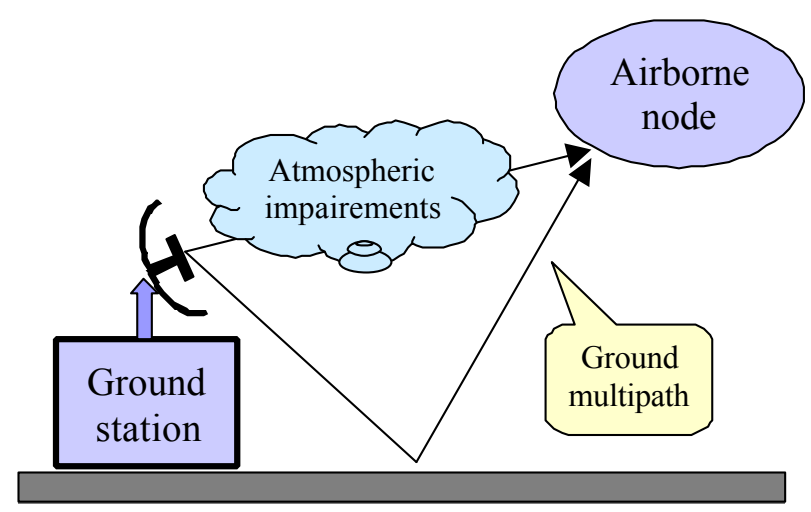

Figure 2. Simplified airborne communication link scenario
Fade events occur usually independently on links 1 and 2 because of their space separation. Thus, the total fade depth on the entire link (between station 1 and station 2) is the same as on links 1 and 2, $F=F_{1}=F_{2}$, assuming that the links 1 and 2 are equal (in the case of unequal links $F=\max \left[F_{1}, F_{2}\right]$ ). The number of fade events on the entire link is twice that of link 1 or 2 . Thus, the outage probability of the entire link is twice that of link 1 or 2 , $P=2 P_{1}=2 P_{2}$ (for unequal links, $\left.P=P_{1}+P_{2}\right)$. It is obvious from the considerations above that the entire link analysis reduces to the analysis of one individual link. Thus, we consider below the simplified scenario shown on Fig. 2.

\section{MODELS FOR FADING PREDICTION}

In general, there are two main contributions to fading [2]: (i) due to ground multipath or due to reflection from ground objects like mountains or hills, and (ii) due to atmospheric impairments (reflection, refraction, ducting etc.). As to the best of our knowledge, there is no specific model for the fade depth prediction in the airborne scenario. There are three types of fading models which are applied to microwave wireless systems:

1. Cellular system fading models, e.g. Rayleigh, Rice or Nakagami models [3, 4]

2. Point-to-point terrestrial LOS link fading models, e.g. Olsen-Segal model [5-7].

3. Satellite link fading models, e.g. ITU-R model $[8,9]$.

The two closest models are the Olsen-Segal model and the ITU-R model for satellite links. However, the airborne link scenario has several specific features, which do not allow to use directly (or with small modifications) one of the models above.

The fading models used for cellular systems are not appropriate for the airborne link because the richness of multipath is much smaller in the latter case and the ground station antenna may have high directivity, which should be accounted for as well. The main source of differences between the airborne link on one side and the terrestrial and satellite links on the other is the difference in geometry and antenna parameters. The specific features of the airborne link is as follows. First, the number of multipath components is small enough when the air-borne antenna is located high enough and the ground antenna clears nearby reflecting objects, with the primary reflection being from the earth surface or some terrain areas like mountains or hills. Secondly, the ground antenna may or may not see the ground depending on the relation between the antenna beamwidth and the path clearance angle. However, as it has been well recognized, the ground reflection gives a substantial contribution to the overall fading level when elevation angle is not large 
enough so the antenna "sees" the ground [10]. This is the case for terrestrial point-to-point LOS links, but may be not the case for the airborne link depending on the path clearance angle and the antenna beamwidth (thus, the antenna pattern must be accounted for). Finally, due to the movement of the air vehicle over large distances, it is very difficult if possible at all to analyze the ground multipath component in the same way as for fixed terrestrial pointto-point links. Thus, a new model is required.

\section{TWO-RAY GROUND MULTIPATH MODEL}

In this Section, we discuss the use of two-ray ground multipath model adapted to more or less realistic scenarios of hilly or mountainous terrain for fade depth prediction when the main contribution is due to the ground reflection and the atmospheric contribution may be neglected, which is the case for frequencies lower than $10 \mathrm{GHz}$ and for elevation angles above few degrees [2]. In all the considerations below, we adopt the geometrical optics approximation and consider singular specular reflection assuming that the size of reflective area is large enough to cover the first Fresnel zone, which constitutes the worstcase fading estimation (out-of-phase combining of LOS and reflected paths is assumed). Fig. 3 depicts the scenario considered.

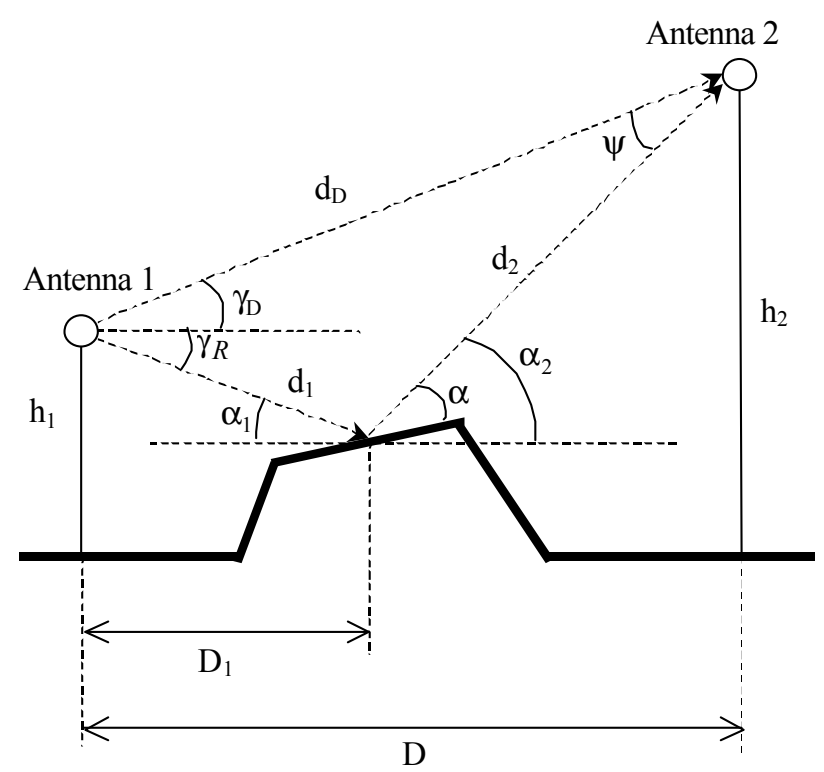

Figure 3. Two-ray multipath propagation scenario for hilly or mountainous terrain

In this scenario, there are two main rays arriving to the airborne antenna 2: the LOS ray and the ground reflected ray. Hence, The total field at the antenna 2 is

$$
E_{\text {total }}=E_{D}+E_{R} \cdot e^{j \Delta \varphi}
$$

where $E_{D}$ and $E_{R}$ are amplitudes of direct (LOS) and reflected rays, respectively, and $\Delta \varphi$ is their phase difference. The minimum received signal level is $E_{\min }=E_{D}-E_{R}$, when the phase difference $\Delta \varphi=\pi$. Consequently, the fade depth $F$ is

$$
F=\left(\frac{E_{D}}{E_{\min }}\right)^{2}=\left(1-\frac{E_{R}}{E_{D}}\right)^{-2}
$$

Using the geometric optics approximation, (5) can be presented in the following form:

$$
F=\left(1-\Gamma \frac{d_{D}}{d_{1}+d_{2}} G_{1}(\gamma) G_{2}(\psi)\right)^{-2}
$$

where $\Gamma$ is the magnitude of the reflection coefficient, and $G_{1}(\gamma)$ is the normalized pattern in the direction of the reflected ray for the ground antenna, $G_{2}(\psi)$ is the normalized pattern of the airborne antenna, $\gamma=\gamma_{D}+\gamma_{R}$ is the path clearance angle, $d_{D}$ is the LOS path length, and $d_{1}+d_{2}$ is the reflected path distance. We assume that the ground antenna, antenna 1 , is steered exactly to the airborne antenna, antenna 2, i.e. $G_{1}(0)=1$, and vice versa, i.e. $G_{2}(0)=1$. The magnitude of the reflection coefficient may be estimated using the classic equations [3]:

$$
\begin{gathered}
\Gamma_{V}=\frac{-\varepsilon_{r} \sin (\alpha)+\sqrt{\varepsilon_{r}-\cos ^{2}(\alpha)}}{\varepsilon_{r} \sin (\alpha)+\sqrt{\varepsilon_{r}-\cos ^{2}(\alpha)}} \\
\Gamma_{H}=\frac{-\sin (\alpha)+\sqrt{\varepsilon_{r}-\cos ^{2}(\alpha)}}{\sin (\alpha)+\sqrt{\varepsilon_{r}-\cos ^{2}(\alpha)}}
\end{gathered}
$$

where $V$ and $H$ stands for vertical and horizontal polarization correspondingly, $\varepsilon_{r}$ is the relative ground permittivity, and $\alpha$ is the local incidence/reflection angle at the reflection point. Note that Eqs. (6) and (7) are general enough to account for the geometry of the problem, the antenna patterns, and the ground permittivity. No detailed path profile is required for this method.

Further we develop simple approximate formulas for practically-important cases, which gives us possibility to get some insight and estimate the fade depth in a simple and fast way (but accurately enough for many practically important problems in the system-level design). We adopt the following assumptions which hold in many cases: $D>>h_{1}, h_{2} ; h_{2}>>h_{1}, D_{l}<<D$. We also assume that the ground antenna "sees" the ground (i.e., $G_{1}(\gamma)=1$ ), i.e. we neglect the ground antenna pattern. The beamwidth of airborne antennas is usually large enough so that 
$G_{2}(\psi)=1$ (note that under the assumptions above $\psi$ will be very small, typically smaller than a degree). Under the assumptions above, $\gamma_{D} \approx \alpha_{2}$ and, consequently,

$$
\alpha=\frac{\alpha_{1}+\alpha_{2}}{2} \approx \frac{\gamma}{2}
$$

Note that $\gamma<<1$ and for a typical ground $\varepsilon_{r} \gg>1$ (for example, for the average ground $\varepsilon_{r} \approx 15$ ). Thus, (7) reduces to

$$
\Gamma_{V} \approx 1-2 \alpha \sqrt{\varepsilon_{r}} ; \quad \Gamma_{H} \approx 1-\frac{2 \alpha}{\sqrt{\varepsilon_{r}}}
$$

Detailed analysis shows that the path length difference effect on the signals' amplitudes can be usually neglected in (6), i.e. $d_{D} \approx d_{1}+d_{2}$ (its effect is approximately 2-3 orders of magnitude smaller then the reflection coefficient effect). Combining (6), (8) and (9), the fade depth can be estimated by remarkably simple formulas:

$$
F_{V} \approx \frac{1}{\gamma^{2} \varepsilon_{r}} ; \quad F_{H} \approx \frac{\varepsilon_{r}}{\gamma^{2}}
$$

Note that in deriving these formulas we assumed that $\gamma>0$ otherwise the LOS path would be obstructed. Eq. (10) may be used provided that $\gamma^{2} \varepsilon_{r} \ll<$ (for vertical polarization) and $\gamma^{2}<<\varepsilon_{r}$ (for horizontal polarization).

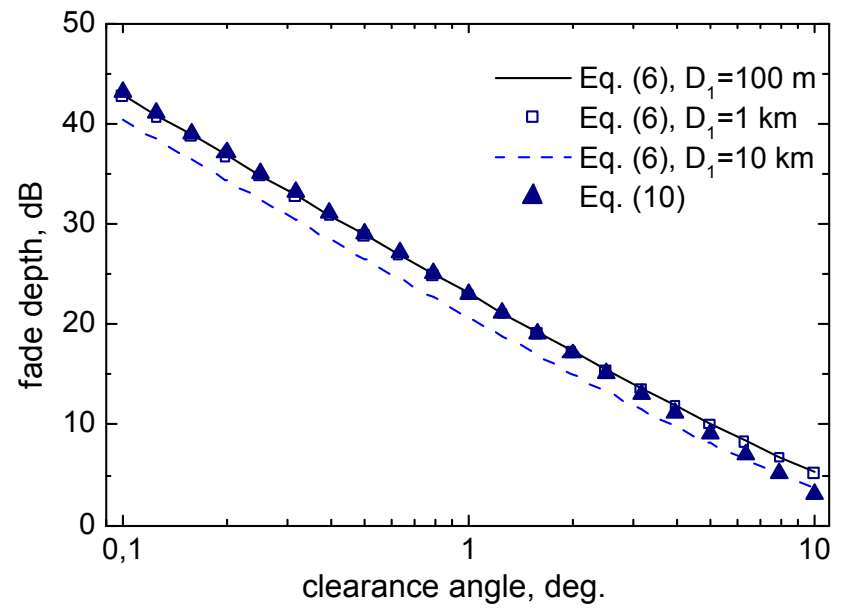

Figure 4. Fade depth versus clearance angle for vertical polarization computed by (6) and (10). $D=40$ $\mathbf{k m}, \mathbf{h}_{\mathbf{1}}=\mathbf{1 0} \mathbf{~ m}, \gamma_{\mathbf{D}}=10^{\circ}, \varepsilon_{r}=16$.

Figure 4 compares the fade depth computed by (6) and (10). As one may see from this figure, (10) provides quite a good approximation to (9) for a wide range of $D_{1}$. As detail analysis shows, the fade depth depends mainly on the clearance angle. Its dependence on the LOS path elevation and the distance to the reflection point is much smaller (provided the clearance angle is fixed). It is interesting to note that if the spherical earth profile is taken into account, the results does almost not change provided that the angles are used as independent variables (as in Fig. 4).

\section{COMPARISON TO OLSEN-SEGAL MODEL}

The Olsen-Segal model $[5,6]$, which has been approved by ITU-R [7], is used for fade depth prediction on terrestrial point-to-point LOS links. In fact, this model is an empirical generalization of the well-known Barnett's model [11] and is based on extensive measurement data available world-wide (for over 240 links in 23 countries). The Olsen-Segal model takes into account both the atmospheric impairments as well as ground reflection. The outage probability for this model is given by:

$$
P=K d^{3.6} f^{0.89}\left(1+10^{3}|\varepsilon|\right)^{-1.4} F^{-1}
$$

where $K$ is the geoclimatic factor, $d$ is the distance $(\mathrm{km}), f$ is the carrier frequency $(\mathrm{GHz}), \varepsilon$ is the path elevation angle (rad.). $P$ can also be considered as the percentage of time in the average worst month when the actual fade depth exceeds $F$. For this model, $F=L_{L} L_{S}$. Note that (11) is valid for high fade depths, $F \geq 15-20 d B$. For shallow fade depths, the interpolation procedure described in $[5,7]$ must be implemented. The other limitations of the model are: (i) $120 \mathrm{MHz} \leq f \leq 37 \mathrm{GHz}$, however the minimum frequency depends strongly on the path length and the minimum path clearance (see [5-7] for more detail), (ii) $10 \mathrm{~km} \leq d \leq 140 \mathrm{~km}$, (iii) $\varepsilon<2^{0}$. Note that the method of calculating the geoclimatic factor world-wide is available as well $[6,7]$. In fact, the Olsen-Segal model (as well as Barnett's model) is a modification of the Rayleigh model to account for frequency, distance, path elevation and geoclimatic conditions. This model predicts fade depth versus elevation angle, which is of great importance for the analysis of the airborne link. However, this model is valid when the elevation angle is less than few degrees, but the elevation angle for the airborne link may be much higher, and it does not account explicitly for antenna pattern. Thus, some additional method is required to predict fade depth for higher elevation angles and to account for the antenna pattern in an explicit way. Such a method has been presented in Section 4 .

Let us now compare the Olsen-Segal model with the results of Section 4. For LOS terrestrial links, the path elevation angle and the clearance angle are approximately the same because the reflection point is usually far away from both antennas, $\varepsilon \approx \gamma$. Hence, for $\gamma$ larger than few milliradians, one obtains from (11):

$$
F \approx C \cdot \gamma^{-1.4}
$$


where $C$ is a constant. Comparing (12) to (10), we see that they give approximately the same dependence of $F$ on $\gamma$. The difference in the power value may be attributed to the ground roughness (which is unaccounted for in (10)) and to the atmospheric fading contribution. Thus, from these considerations we may speculate that a substantial contribution to fading in (11) is due to ground or atmospheric reflection. This may be, as to the best of the authors' knowledge - for the first time, a theoretical justification of the path elevation factor in the Olsen-Segal model. Some additional studies are required to account for ground roughness, atmospheric fading contribution and the effect of antenna pattern (in the Olsen-Segal model).

\section{HYBRID APPROACH}

The two ray multipath model discussed in Section 4 can be applied to airborne links when the main contribution to fading is due to ground multipath because this model does not account for the atmospheric impairments. The OlsenSegal model, being empirical in nature, accounts for all the fading mechanisms encountered in the terrestrial LOS links. However, the airborne scenario is quite different from that of the terrestrial links. Besides, the Olsen-Segal model is valid for low elevation angles (below app. 2 degrees). The ITU-R model for fade depth prediction on satellite links $[8,9]$ is valid for wide range of elevation angles but the airborne link scenario is different from the satellite links scenario (antenna beamwidth, path geometry, frequency etc.).

To overcome these difficulties, we propose a hybrid approach to fade depth estimation on the airborne links. It consists of the following steps:

1. The Olsen-Segal model is used for marginal fade depth estimation, including both atmospheric and ground-reflected components.

2. The ITU-R model for earth-to-space path is used for marginal fading estimation, but this includes only the atmospheric component.

3. The two-ray multipath model of Section 4 is used for fading estimation due to ground reflection.

4. The total fade depth is considered to be the maximum of steps 1-3 (due to statistical independence of ground and atmospheric contributions)

A detailed comparative analysis of the predictions of the Olsen-Segal and ITU-R models allows drawing the following conclusions:

- The fade level is very high (up to 20-30 dB) for small elevations (below few degrees) and drops down very rapidly (to few dBs) for higher elevations. This is believed to be due to the very narrow-beam antennas used in the applications covered by these models (few degrees for the $1^{\text {st }}$ model, and fractions of a degree for $2^{\text {nd }}$ model).

- Ground reflection component is an important contributor to fading, especially for small elevations.

- The fade levels for higher elevations (above few degrees) predicted by both models are roughly the same (few dBs). This is believed to be a pure contribution of atmospheric fading (ground component is excluded due to high antenna directivity).

It should be noted that the Oslen-Segal model is limited to elevations up to 2 degrees. However, as the detail comparative analysis shows, the fade depth decreases above this elevation very slowly (few dBs only). One expects that the actual fade depth decreases with elevation as well. Thus, it could be lower than the Olsen-Segal model predictions, but certainly not higher. Hence, the Olsen-Segal model provides a good conservative estimation of the atmospheric fading at these elevations.

In fact, the hybrid approach is a combination of two well-known empirical models with the theoretical model. Some additional theoretical analysis and filed measurements are required to validate this approach.

\section{CONCLUSIONS}

Fade depth prediction for the power budget analysis of airborne communication links has been discussed in this paper. At the moment, there is no fading model for this particular scenario. The two closest models are the OlsenSegal model and the ITU-R model for satellite links. However, they cannot be directly applied to the airborne link scenario. The two-ray ground multipath model adapted to a realistic scenario of hilly or mountainous terrain has been used for fade depth prediction on airborne links in this paper.

The comparison of the two-ray model with the OlsenSegal model shows that they predict roughly the same fade depth dependence on the elevation angle. This may be considered, as to the best of our knowledge - for the first time, as a theoretical justification of the path elevation factor in the Olsen-Segal model.

The hybrid approach has been proposed to account for the atmospheric as well as ground reflection components of the fading.

The two-ray model above and the flight dynamics parameters were further used to propose a time-varying dispersive channel model for an airborne communication system [12]. A channel emulator programmed accordingly was then used to simulate the airborne communication system and obtain parametric performance results.

\section{ACKNOWLEDGEMENTS}

We would like to thank Prof. N. Batani, Prof. J. Belzile, and Mr. Girard for many fruitful discussions. 


\section{REFERENCES}

1. D.R. Smith, Digital Transmission Systems, Van Nostrand Reinhold, New York, 1993.

2. R.L. Freeman, Radio System Design for Telecommunications, John Wiley \& Sons, 1997.

3. D. Parson, The Mobile Radio Propagation Channel, U.K.: Pentech, 1992.

4. K. Siwiak, Radiowave Propagation and Antennas for Personal Communications, Artech House, Boston, 1995

5. R.L. Olsen, B. Segal, New Techniques for Predicting the Multipath Fading Distribution on VHF/UHF/SHF Terrestrial Line-of-Sight Links in Canada, Canadian Journal of Electrical and Computer Engineering, v. 17, N. 1, 1992, pp. 11-23.

6. R.L. Olsen, T. Tjelta, Worldwide Techniques for Predicting the Multipath Fading Distribution on Terrestrial LOS Links: Background and Results of Tests, IEEE Trans. AP, v. 47, N. 1, Jan. 1999, pp. 157-170.

7. Recommendation ITU-R P.530.5, "Propagation Data and Prediction Methods Required for the Design of
Terrestrial Line-of-Sight Systems", Geneva, Switzerland, 1999.

8. L.J. Ippolito Jr., Satellite Transmission Impairments, in J.D. Gibson (Ed.), "Communications Handbook", Boca Raton, Flor.: CRC Press , 1997.

9. Recommendation ITU-R P.618-6, "Propagation Data and Prediction Methods Required for the Design of EarthSpace Telecommunication Systems", Geneva, Switzerland, 1999.

10.R.L. Olsen, L. Martin, T. Tjelta, A Review of the Role of Surface Reflection in Multipath Propagation Over Terrestrial Microwave Links, NATO/AGARD Conf. On Terrestrial Propagation Characteristics in Modern Systems of Communications, Surveillance, Guidance and Control, N. CP-407, Nov. 1987, pp. 2/1-1/23.

11.W.T. Barnett, Multipath Propagation at 4, 6, and 11 GHz, The Bell System Technical Journal, v. 51, N. 2, Feb. 1972, pp. 321-361.

12. A. Kouki et al, Airborne Communication Node Study, Technical Report, Ecole de Technologie Superieure, Montreal, July 2000. 